\title{
Borrelia burgdorferi Manipulates Innate and Adaptive Immunity to Establish Persistence in Rodent Reservoir Hosts
}

\author{
Karen E. Tracy ${ }^{1,2}$ and Nicole Baumgarth ${ }^{1,2,3 *}$ \\ ${ }^{1}$ Graduate Group in Immunology, University of California Davis, Davis, CA, USA, ${ }^{2}$ Center for Comparative Medicine, \\ University of California Davis, Davis, CA, USA, ${ }^{3}$ Department of Pathology, Microbiology and Immunology, University of \\ California Davis, Davis, CA, USA
}

OPEN ACCESS

Edited by:

Monica E. Embers,

Tulane University, USA

Reviewed by:

Peter Kraiczy,

Goethe University Frankfurt, Germany

Robin Stephens,

University of Texas Medical

Branch, USA

${ }^{*}$ Correspondence:

Nicole Baumgarth

nbaumgarth@ucdavis.edu

Specialty section: This article was submitted to Microbial Immunology, a section of the journal

Frontiers in Immunology

Received: 06 November 2016 Accepted: 25 January 2017 Published: 20 February 2017

Citation:

Tracy KE and Baumgarth N (2017) Borrelia burgdorferi Manipulates Innate and Adaptive Immunity to Establish Persistence in Rodent Reservoir Hosts.

Front. Immunol. 8:116. doi: 10.3389/fimmu.2017.00116
Borrelia burgdorferi sensu lato species complex is capable of establishing persistent infections in a wide variety of species, particularly rodents. Infection is asymptomatic or mild in most reservoir host species, indicating successful co-evolution of the pathogen with its natural hosts. However, infected humans and other incidental hosts can develop Lyme disease, a serious inflammatory syndrome characterized by tissue inflammation of joints, heart, muscles, skin, and CNS. Although B. burgdorferi infection induces both innate and adaptive immune responses, they are ultimately ineffective in clearing the infection from reservoir hosts, leading to bacterial persistence. Here, we review some mechanisms by which $B$. burgdorferi evades the immune system of the rodent host, focusing in particular on the effects of innate immune mechanisms and recent findings suggesting that T-dependent B cell responses are subverted during infection. A better understanding of the mechanisms causing persistence in rodents may help to increase our understanding of the pathogenesis of Lyme disease and ultimately aid in the development of therapies that support effective clearance of the bacterial infection by the host's immune system.

Keywords: immune evasion, immune exhaustion, germinal center, complement inhibition, persistent infection, lyme disease

\section{INTRODUCTION}

Borrelia burgdorferi sensu lato is a species complex of spirochetal bacteria that infects a wide variety of mammals, birds, and reptiles. It includes, most notably, Borrelia burgdorferi sensu stricto (Borrelia burgdorferi), Borrelia afzelii, and Borrelia garinii (1), the three most prevalent causative agents of Lyme disease in humans (2). According to CDC reports, Lyme disease caused by $B$. burgdorferi is currently the most common vector-borne disease in the United States (3). In Europe, infections with B. afzelii and B. garinii are more prevalent than those with B. burgdorferi. In Asia, of the three primary disease-causing species, only B. afzelii and B. garinii are present (4). Other Borrelia species, including Borrelia bavariensis and Borrelia spielmanii, can also cause infection and disease in humans (5). Thus, B. burgdorferi sensu lato infections are an important global public health concern.

The bacteria are transmitted between hosts by ticks of the genus Ixodes. B. burgdorferi infection is therefore only common in areas where these vector species thrive. Deer mice (Peromyscus 
leucopus) are often considered the major reservoir host in the United States (6). In mice, spirochetes form persistent, nonresolving infections (7). However, these infections do not cause noticeable manifestations of disease in P. leucopus or certain common laboratory strains of mice [Mus musculus] (8-10). This suggests that $B$. burgdorferi has developed immune evasion strategies that allow it to persist in the face of a mammalian immune system. Such mechanisms may be the products of co-evolution with reservoir hosts, minimizing host disease manifestations while maximizing bacterial growth and transmission.

Laboratory mouse studies have been used to better understand B. burgdorferi pathogenesis in humans (11), just as they have for many other disease processes. They also provide an opportunity for better understanding the amplification and spread of B. burgdorferi in wild rodents, which in turn affects the infection risk of humans in endemic areas. Furthermore, B. burgdorferi in the mouse is an excellent model system for better understanding the mechanisms by which certain pathogens can achieve persistence in immunocompetent hosts $(12,13)$. Here, we summarize known mechanisms by which $B$. burgdorferi circumvents innate and adaptive immune responses to establish lifelong persistence in the mouse host. We examine interference with both the innate and adaptive immune systems. We emphasize bacterial persistence over disease and tissue pathology, because persistence of $B$. burgdorferi in wild rodents is a prerequisite for human infections.

\section{EVIDENCE FOR CO-EVOLUTION OF B. burgdorferi WITH VERTEBRATE HOSTS AND VARIATION IN HOST RESPONSES}

A reservoir host is one in which a particular pathogen can survive with minimal affect to that host. The pathogen can subsequently be transmitted to other species that may experience ill effects. Incidental, or "dead-end," hosts do not facilitate transmission of the pathogen to another host, but often experience manifestations of disease. This is the case for B. burgdorferi, where the reservoir hosts, including wild rodents [Peromyscus spp. (14, 15)] and passerine birds [canary finches (16)], are largely asymptomatically infected, whereas incidental hosts like humans can sometimes suffer a severe array of diseases, including arthritis, carditis, and skin and neurological disease manifestations $(11,17)$.

As outlined earlier, Borrelia species require both an invertebrate vector (ticks of the Ixodes genus) and a vertebrate host to complete their life cycle (11). Many ecological and evolutionary factors affect prevalence, persistence, and disease development by Borrelia infections in both vector and host. These factors include population dynamics, dispersal/migration, and evolution of all three players, as well as environmental landscape and climate (4). Further complicating the situation is the fact that both spirochetes (B. burgdorferi sensu lato) and the vectors (Ixodes ricinus species complex) are part of large species complexes, which have their own unique evolutionary patterns (4). Therefore, individual effects on Borrelia persistence can be difficult to untangle.

The existence of co-evolution with reservoir, but not incidental hosts like humans, remains to be rigorously tested with population genomics approaches (18). However, current evidence supports this hypothesis. For example, differential resistance to complement, an important and evolutionary conserved innate immune defense mechanism, has been suggested to drive host specializations of various Borrelia species to mammals, birds, and reptiles (19). In addition, there is evidence that selection acts on $B$. burgdorferi, within the reservoir host, to generate sequence diversity and polymorphisms relevant to virulence (20).

Co-evolution of host and pathogen must achieve a balance between the induction of immune mechanisms that reduce pathogen burden and pathogen-induced diseases, without clearing the infection. How this is achieved is incompletely understood. The continued presence of the pathogen in its host should provide ongoing triggers for both innate and adaptive immune response induction. Current research suggests that the immune system has important immune checkpoints that regulate immune responses, leading to a state of "immune exhaustion" during chronic infections. The process of immune exhaustion was first identified in chronic LCMV infection (21), leading to the discovery of distinct functions manifested in distinct transcriptional profiles of "exhausted T cells" in both mice and humans in response to a variety of chronic infections (22-25). The state of immune exhaustion goes beyond suppression of the T cell compartment alone, encompassing alterations in both innate and adaptive immune responses [reviewed in Ref. (26)]. While immune exhaustion has not been studied in the context of persistent infection with B. burgdorferi, it is conceivable that this process is also involved in the establishment of Borrelia persistence in its natural reservoir host. Indeed, mounting evidence suggest that the adaptive immune response is suppressed during B. burgdorferi infection.

\section{EVIDENCE OF B. burgdorferi PERSISTENCE}

Persistent infection of reservoir hosts increases the odds that $B$. burgdorferi will be passed on to new hosts. It is challenging to distinguish between high prevalence of reinfection and/or true persistence occurring in the natural habitat of a reservoir host, such as the white-footed mouse P. leucopus $(14,15,27)$. Experimental infections of $P$. leucopus have confirmed, however, that persistence of $B$. burgdorferi can occur, at least in laboratory settings (20). Experimental evidence also shows that B. burgdorferi persists in various laboratory mouse strains, either with or without disease manifestations, depending on the strain used ( 7 , 8). Persistence after experimental infection was also observed in chipmunks (28), dogs (29), canaries (16), and non-human primates $(30,31)$. Figure 1 summarizes mechanisms that can support Borrelia persistence.

\section{INTERFERENCE OF B. burgdorferi WITH THE INNATE IMMUNE SYSTEM}

Ticks provide the first defenses for $B$. burgdorferi against the innate immune system of the mammalian host. B. burgdorferi is transmitted from the tick to the mammalian host within $<16-72 \mathrm{~h}$ after onset of tick feeding (32), where it encounters 


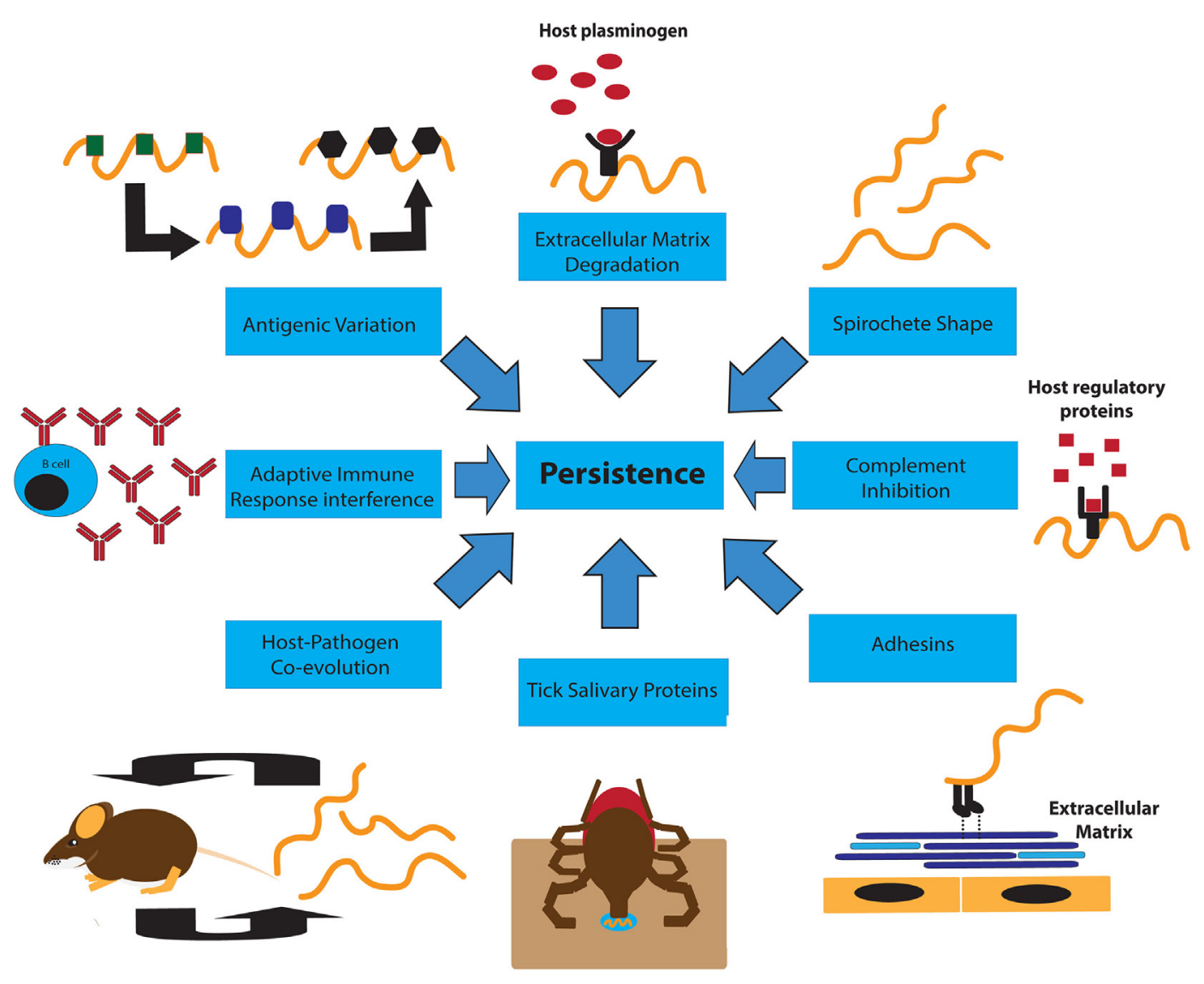

FIGURE 1 | An overview diagram of factors contributing to persistence of Borrelia burgdorferi in rodent hosts. Shown are eight bacterial characteristics and mechanisms that $B$. burgdorferi may use to establish persistence in the rodent host: Spirochete shape (38, 123), antigenic variation and changes in gene expression $(77,83,89)$, plasminogen binding and destruction of the extracellular matrix (52), interference with the adaptive immune response (69, 74, 75), host-pathogen co-evolution $(20,124)$, tick salivary protein-mediated immunosuppression (34), adhesins allowing entrance into the vasculature and tissues (49, 50), and interference with complement via CRASPs, and BBK32 (45, 60, 62, 64).

host defenses present in the skin. Tick salivary proteins play a role in suppressing the host immune system as long as the tick vector is attached. This includes the inhibition of vigorous activation of skin-resident macrophages and dendritic cells, including the suppression of cytokine and chemokine production, and inhibition of granulocyte recruitment to the site of the tick bite [reviewed in Ref. $(33,34)]$. Multiple tick salivary proteins are known to interfere with the activation of the alternative complement pathway, potentially further supporting pathogen transmission [reviewed in Ref. (33)]. Mast cells also appear to be direct targets of salivary protein-mediated immune suppression (35). Inhibition of mast cells was facilitated through secretion of the salivary protein sialostatin $\mathrm{L}$, which was shown to inhibit the induction of IL-9 production in the skin. IL-9 is known as an important regulator of pathogen-induced immune responses (35-37).

The morphology of spirochetes facilitates versatile motility that is predicted to play a role in the dissemination and persistence of Leptospira, Treponema, and Borrelia genera $(38,39)$. $B$. burgdorferi itself also expresses known immunomodulatory surface proteins, which help to modulate immune responses of the host. These proteins, specifically lipoproteins, have been studied extensively, and their wide variety of functions is reviewed elsewhere (40). We focus here on known functions of some proteins that are likely to contribute to persistence of B. burgdorferi in the rodent host (Table 1).

One important group of such proteins is the adhesins, which mimic host integrins, molecules that facilitate attachment and migration. Thus, B. burgdorferi seems to subvert existing mechanisms regulating immune cell migration for its benefit. Adhesins are an important category of bacterial virulence factors that protect bacteria from clearance by physical forces such as mucociliary clearance, facilitate homing to and entrance into host tissues that act as important pathogen niches and trigger signaling events in host cells (41). BBK32 is one such adhesin. At the initial site of infection, BBK32 was shown to create "catch bonds" that slow bacterial movement enough for flagella-driven entrance of B. burgdorferi into the vasculature (42). This helped to explain why bacterial loads in the blood are higher when BBK32 is expressed (43). Once in the blood, BBK32 seems to help B. burgdorferi to target joint tissues for colonization via binding to glycosaminoglycan (44). However, recent studies suggested 
TABLE 1 | B. burgdorferi's interface with mammalian hosts and its effect on Borrelia survival.

\begin{tabular}{|c|c|c|}
\hline Immune response modulators & Effects & Reference \\
\hline Co-evolution of $B$. burgdorferi and its hosts & $\begin{array}{l}\text { Host specialization and evolution of virulence- and infectivity-associated } \\
\text { genes }\end{array}$ & $(19,20)$ \\
\hline Tick salivary proteins & Suppression of pro-inflammatory responses in the host & $(33,34,125)$ \\
\hline Spirochete morphology and motility & Increase in B. burgdorferi dissemination and persistence & $(38,39)$ \\
\hline B. burgdorferi adhesins & Interactions with host tissues, contributing to dissemination and persistence & $(49,50)$ \\
\hline Host interactive proteins & $\begin{array}{l}\text { Binding to host enzymes, such as plasmin/plasminogen; facilitates } \\
\text { extracellular matrix degradation }\end{array}$ & $(50,52)$ \\
\hline CRASPS & Decreased and inhibited complement activation & $(60,62,63)$ \\
\hline Modulation of protein expression & $\begin{array}{l}\text { Adaptation to host, downregulation of immunogenic proteins, and antigenic } \\
\text { variation }\end{array}$ & $(79,80)$ \\
\hline Inappropriate macrophage activation & Extracellular matrix degradation & $(54)$ \\
\hline Antibodies with lgM-skewed isotype profile and of low affinity & Decreased antibody response quality which may contribute to persistence & $\begin{array}{l}\text { (see text footnote) } \\
(73-75)\end{array}$ \\
\hline $\begin{array}{l}\text { Loss of demarcated T and B cell zones in secondary } \\
\text { lymphoid tissues and collapse of germinal centers }\end{array}$ & $\begin{array}{l}\text { Reduced antibody class switch recombination and somatic affinity maturation. } \\
\text { Failure to induce long-lived plasma cells and memory B cells in a timely manner }\end{array}$ & $(69,73,74,110)$ \\
\hline
\end{tabular}

that BBK32 has additional, complement-inhibitory, functions (45). Specifically, it was found to bind to the C1 complex and thereby to inhibit the classical pathway of complement activation, i.e., antibody-mediated bacterial clearance (45).

Another adhesion protein of B. burgdorferi, p66, was shown to interact with ligands on the host vasculature to facilitate $B$. burgdorferi extravasation from blood vessels into tissues (46). $B$. burgdorferi also expresses two decorin-binding proteins (Dbp), DbpA and DbpB. Surface expression of these proteins seems to increase the level of tissue colonization (47). They seem to support persistence of $B$. burgdorferi in tissues that express high levels of decorin (joint tissue and skin) (48). Further details about $B$. burgdorferi-expressed adhesins have been summarized in recent reviews $(49,50)$.

Once bacteria have gained access to a specific organ or tissue, they require proteases that degrade the extracellular matrix (ECM), enabling the bacteria to move between cells deeper into the tissues. This is particularly important for B. burgdorferi, which targets ECM-rich connective tissues $(50,51)$. The genome of $B$. burgdorferi does not seem to contain any known ECM-degrading proteases. Instead B. burgdorferi is able to bind host urokinase and plasminogen, a multifunctional serum protein that can initiate ECM and fibrinogen degradation (52). Plasminogen can also inhibit complement activation and promote complement degradation (53).

Furthermore, B. burgdorferi can induce host cells such as macrophages to secrete matrix metalloproteases (MMPs), particularly the gelatinase MMP-9, via TLR2-mediated immune activation (54). MMP-9 was shown to be selectively upregulated in erythema migrans skin lesions during acute B. burgdorferi infections of humans (55) and is thought to help B. burgdorferi tissue dissemination by enabling the degradation of the ECM. However, studies by $\mathrm{Hu}$ and colleagues recently demonstrated that MMP-9 expression is not required for B. burgdorferi dissemination. Instead, it regulated the influx of inflammatory cells, and thereby Lyme arthritis, indirectly by the degradation of collagen in joints (56). Plasminogen-binding proteins have not been shown to increase $B$. burgdorferi persistence per se, but they do facilitate the entrance of $B$. burgdorferi into the ECM of tissues, where bacteria maybe protected from innate immune response mechanisms such as complement-mediated degradation and/or neutralization by early-induced IgM antibodies. ${ }^{1}$

\section{Interference of $B$. burgdorferi with the Complement System}

As mentioned earlier, inhibition of the complement system is an important immune evasion strategy employed by many pathogens, including B. burgdorferi (57-59). B. burgdorferi proteins that interfere with complement activation allow for survival and dissemination of the pathogen from the initial site of infection (60).

The complement system consists of an evolutionarily highly conserved family of proteins that are found in all body fluids and serve three main functions during infection: trigger inflammation, opsonize pathogens, and form the "membrane attack complex" (formation of a pore in the cell membrane that causes cell lysis). Classical, alternative, and lectin are the three distinct pathways by which complement-mediated signaling and bacterial killing can be initiated [described in more detail in Ref. (61)]. Independent of the initial trigger, all pathways lead to the formation of a protease, the C3 convertase, which cleaves the complement component $\mathrm{C} 3$ into its activated components $\mathrm{C} 3 \mathrm{a}$ (an inflammatory mediator) and C3b (an opsonin and immune stimulatory protein). The complement component $\mathrm{C} 3 \mathrm{~b}$ can also form the $\mathrm{C} 5$ convertase, another protease that cleaves complement component $\mathrm{C} 5$ into $\mathrm{C} 5 \mathrm{a}$ and $\mathrm{C} 5 \mathrm{~b}$, leading to the formation

${ }^{1}$ Hastey CJ, Elsner RA, Olsen KJ, Tunev SS, Escobar ED, Barthold SW, et al. Borrelia burgdorferi infection-induced IgM controls bacteremia but not bacterial dissemination or tissue burden. (submitted). 
of the bactericidal "membrane attack complex" (61). C5a, like $\mathrm{C} 3 \mathrm{a}$, is a strong inducer of inflammation. During the process of complement activation, another complement component, C4, is cleaved into $\mathrm{C} 4 \mathrm{a}$ and $\mathrm{C} 4 \mathrm{~b}$. The latter also acts as an opsonin. Because of these multiple and highly pro-inflammatory effects, systemic activation of complement can cause septic shock if not appropriately regulated. Regulators of overshooting complement activation include components of the complement system itself: the Factor $\mathrm{H}$ family proteins and the $\mathrm{C} 4 \mathrm{~b}$-binding protein. These proteins inhibit complement activation by a variety of mechanisms, including by accelerating the decay of $\mathrm{C} 3$ convertases, thus interrupting the complement activation cascade.

Borrelia burgdorferi has evolved complex mechanisms to evade complement-mediated killing by binding to the inhibitory host-regulatory factors [reviewed in Ref. $(60,62)$ ]. B. burgdorferi expresses a diverse family of complement regulator-acquiring surface proteins, which recruit Factor $\mathrm{H}$ family proteins [reviewed in Ref. (63)]. Factor $\mathrm{H}$ and its relatives primarily inhibit activation of the alternative complement pathway. More recently, it was discovered that B. burgdorferi also binds to host C4-binding proteins, which primarily inhibit activation of the classical and lectin pathways (64). And, as outlined earlier, BBK32 seems to inhibit the classical pathway of complement activation via binding to the C1 complex (45). Thus, B. burgdorferi seems to target all three activation pathways of the complement cascade. The effects of complement inhibition on adaptive immune responses are outlined below.

\section{INTERFERENCE OF B. burgdorferi WITH THE INDUCTION OF ADAPTIVE HUMORAL IMMUNE RESPONSES}

High levels of $B$. burgdorferi antigen-specific antibodies are produced during infection, and they have the capacity to prevent reinfection with the same $B$. burgdorferi strains (65-67). The antibody response also results in reduction, but not elimination, of B. burgdorferi from tissues (68). Both T-independent and T-dependent antigens are targeted by the humoral immune response, representing a wide variety of surface proteins with different functions $(20,67,69)$. How then does B. burgdorferi avoid antibody-mediated clearance?

\section{B. burgdorferi-Induced Humoral Immunity}

The strong production of $B$. burgdorferi antigen-specific antibodies, as well as the strong increases in B. burgdorferi tissue load in SCID mice and B cell-deficient mice compared to wild-type controls $(70,71)$, has long been considered evidence of a robust and effective $B$ cell response against B. burgdorferi infection. However, while the data provide clear evidence that B cells play an important role in the control of B. burgdorferi infection, this does not mean that these responses are optimally induced. Considering that $B$. burgdorferi infection results in persistent infections of mice despite these robust antibody responses, it is important to also consider the quality of the B. burgdorferispecific antibody response. Several factors are known to affect the efficacy of humoral immune responses, including the epitope specificity of the induced antibodies, their immunoglobulin isotype profile, binding avidity to their cognate antigens, and various posttranslational modifications that can affect their effector functions $(6,20)$.

IgM is the first antibody isotype secreted during an immune response. It is important in controlling bacteremia and in activating the classical complement pathway. Immunoglobulin class switch recombination (CSR) to IgG typically occurs soon after an infection, and the four subtypes of murine IgG work together effectively to clear most pathogens (72). However, during B. burgdorferi infection, serum IgM levels remain high throughout infection (see text footnote). Moreover, hybridomas generated from lymph nodes of mice on days 8 and 18 postinfection showed that nearly half of $B$. burgdorferi antigen-specific cells were producing IgM, and the ratio of IgM to IgG never significantly changed throughout the infection (see text footnote) (73). The strong and ongoing production of IgM cannot be explained entirely by a predominance of T-independent responses, because depletion of CD4 T cells decreased the number of IgM-antibody-secreting cells (ASCs) (74). Thus, infection with B. burgdorferi induces an antibody response that is characterized by the continued production of IgM and IgG. Further studies will need to determine whether the strong production of IgM is evidence of a strong beneficial immune response or whether B. burgdorferi might be subverting the $B$ cell response to generate this unusual Ig-isotype profile. Our studies have failed to find any beneficial effect of IgM on control of bacterial dissemination or B. burgdorferi tissue loads (see text footnote).

Given the important role of $\mathrm{T}$ cells in the regulation of CSR by $\mathrm{B}$ cells, the data may indicate a deficiency in $\mathrm{T}$ helper cell activation and/or functionality. In vitro data indeed provided some evidence that the CD4 T helper cell response induced by $B$. burgdorferi infection is distinct in function from that of CD4 T cells induced by immunizing mice with inactivated $B$. burgdorferi (75). T-dependent B cell responses usually also result in significant affinity maturation, i.e., an increase in the binding avidity of antibodies to their cognate antigens over time. Measuring antibody avidities of serum antibodies to a representative T-dependent antigen on B. burgdorferi N40, arthritis-related protein (Arp), however, failed to provide evidence for affinity maturation in the serum response to B. burgdorferi (75). Instead, we found that the binding avidity of the serum antibodies to Arp initially increased for the first 6 weeks of infection, but then peaked and steadily decreased thereafter to levels seen at the beginning of the infection. The rate of drop in antibody affinity was consistent with the normal half-life and decays kinetics of serum antibodies (75), suggesting that the ASC that generated the higher-affinity antibodies were short-lived.

Both CSR and hyperaffinity maturation of antibodies are T-dependent processes that usually occur in germinal center (GC) reactions in secondary lymphoid tissues. As we will outline below in more detail, we noted a collapse of the GC responses that coincided with the peak and then reversal of the antibody avidities. The data strongly suggest that the T-dependent GC responses are not fully functional during $B$. burgdorferi infection. The fact that immunization with inactivated $B$. burgdorferi infection resulted in robust GC responses suggests that exposure 
to live B. burgdorferi results in a subversion of the B cell response $(73,74)$. On the basis of these data, we propose that although present in large quantities, the B cell responses to and functionality of the serum antibody response to B. burgdorferi are suboptimal, enabling B. burgdorferi persistence, while also controlling $B$. burgdorferi tissue loads and thus overwhelming infection. Alterations in the $\mathrm{B}$ cell response quality and/or antibody functionality could provide a powerful immune evasion strategy for bacteria that are clearly susceptible to antibody-mediated immune clearance mechanisms $(65,66,70,71,76,77)$.

\section{Modification of B. burgdorferi Outer Surface Protein Expression}

Although B. burgdorferi surface proteins have many functions in evading recognition by the host immune system, they are also antigens that trigger antibody responses. Additional immune evasion strategies therefore likely exist that inhibit recognition and antibody-mediated clearance of B. burgdorferi.

Borrelia burgdorferi is known to undergo major protein expression changes over the course of its life cycle, including expression of outer surface proteins. During transmission from the tick to a mammalian host, environmental signals trigger extensive global changes in gene expression $(78,79)$. Changes in surface protein expression also occur within a mammalian host during the course of infection. At least some proteins that trigger strong antibody responses are downregulated during the chronic phase of infection (80). One important example is OspC, a T-dependent antigen that is essential for initial colonization of mammalian hosts. Shortly after infection, this lipoprotein is no longer required, and if not downregulated triggers a strong and effective antibody response. However, expression is usually rapidly lost upon infection (81-84). This appears to be an effective immune evasion strategy, because constitutive expression of OspC prevents spirochete persistence, whereas bacteria that successfully downregulate OspC outcompete expressors in vivo $(76,85)$.

Antigenic variation is a process by which a pathogen varies the sequence of an expressed protein to avoid the deleterious effects of antibodies raised against it. Antibody-target switching has been demonstrated as an effective immune evasion strategy for numerous pathogens, including the closely related relapsing fever pathogen Borrelia hermsii (86-88). The B. burgdorferi genome contains the variable surface antigen $\mathrm{E}\left(v l_{s} E\right)$ locus, which has received much attention as a major immunodominant surface protein of B. burgdorferi that undergoes extensive and rapid antigenic variation in mammalian hosts [reviewed in Ref. (89)]. In B. burgdorferi strain B31, VlsE recombination seems to be critical for $B$. burgdorferi persistence and the ability of $B$. burgdorferi to reinfect a host following antibiotic treatment (77, 90-92). It is interesting to note that vlsE recombination also occurs in antibody-deficient SCID mice, but not in vitro, suggesting that host triggers other than the antibody response direct this process (93). While it seems that a lack of variation of vlsE results in the rapid clearance of B. burgdorferi, it is less clear whether this process alone can explain the effective evasion of $B$. burgdorferi from antibody-mediated clearance. Recently, mathematical models have been put forward to suggest that a strongly immunodominant variable surface protein may prolong immune responses long enough to drive immune exhaustion (94), an intriguing idea that requires testing in the context of B. burgdorferi infection.

Finally, extensive genetic variation exists between the various Borrelia species and between individual bacteria (95-98). This allows adaptation to selective pressure from the host immune system, as well as to larger ecological niches, allowing strains to become fitter in a given geographic area (95). It also applies during the course of a chronic infection, as bacterial variants compete with each other $(85,99)$. Variation between strains is large enough in the context of the antibody response that different strains can infect the same mouse (100). Reinfection with the same strain has been tested in experimental settings after antibiotic treatment of mice. Studies by Piesman et al. and by Elsner et al. suggested that antibody-mediated protection wanes over time, even to the same strain of B. burgdorferi (69, 101). Given that long-term antibody production is usually induced following an infection, facilitated by the development of long-lived plasma cells that migrate to and then reside in the bone marrow $(102,103)$, the data further suggest that the antibody response to $B$. burgdorferi infection lacks some of these key components of successful humoral responses. It can be expected that each of the above outlined immune evasion mechanisms is unlikely to be solely responsible for persistence; instead they may act in concert. Whether these multiple apparent strategies represent redundant or synergistic effects remains to be established.

\section{B. burgdorferi Modulates the Adaptive Immune Response}

Modeling of population dynamics supports the establishment of equilibrium between $B$. burgdorferi and the host's adaptive immune response (104). This is consistent with recent findings suggesting that the humoral immune response and, more specifically, the T-dependent B cell responses are a particular target of $B$. burgdorferi-induced immune suppression.

After escaping the site of infection, B. burgdorferi disseminates to other tissues via blood and lymph $(43,105)$. Mechanisms of entrance into the blood are better studied, but live B. burgdorferi is also capable of entering, persisting in, and traveling through the lymphatics $(73,105)$. Dissemination via the lymphatics has been described in other infections as an effective technique for immune evasion due to the specialized cell populations and altered immune milieu in these vessels compared to the bloodstream (106-108). Shortly after infection with cultured or host-adapted spirochetes (HAS), live B. burgdorferi enter lymph nodes draining the site of infection, resulting in massive lymph node enlargement $(73,109)$.

In secondary lymphoid tissues such as the lymph nodes, naïve $\mathrm{T}$ cells encounter antigens for the first time, and activated antigen-specific $B$ cells receive the signals they need to proliferate. During B. burgdorferi infection, the presence of live spirochetes seems to interfere with these processes. Between days 5 and 7 postinfection of C57BL/6 mice with HAS, the separation of $\mathrm{T}$ cell zones and B cell follicles completely was lost within the draining lymph nodes. This degradation was not seen in mice that were inoculated with heat-inactivated 
spirochetes, suggesting that an active bacterial process causes the disruption of the normal immune response of the host (73, $74,110)$. Interestingly, similar observations have been made also following infections with other pathogens, including infections with Plasmodium and Salmonella $(111,112)$. While during Salmonella infection lymph node architecture disruption was dependent on TLR4-mediated signaling (112), the observed changes during $B$. burgdorferi infection were independent of MyD88 and TRIF signaling (110). Given the importance of T and $\mathrm{B}$ cell trafficking within the lymph nodes for initiating and maintaining $\mathrm{T}-\mathrm{B}$ interaction and immune response regulation, the data suggest that multiple pathogens, including B. burgdorferi, may have evolved strategies to interfere with the earliest processes of adaptive immune induction.

Furthermore, the presence of live (but not heat-killed) $B$. burgdorferi caused a disproportionate recruitment and proliferation of naïve follicular B cells, but not CD4 T cells, to the affected lymph nodes. This appeared to be a separately controlled process, but one also mediated specifically by live bacteria. B cell recruitment and/or retention required signaling through the type I interferon receptor by non-hematopoietic cells, likely lymph node stromal cell compartments (110). The resulting accumulation of large numbers of B cells in the lymph nodes explains the lymphadenopathy that is also observed in infected humans and dogs $(113,114)$. This pattern of lymph node architecture disruption followed by influx/retention of B cells is recapitulated later in more distant lymph nodes as they are infiltrated by the migrating spirochetes $(73,110)$.

The data support the concept that B. burgdorferi interferes with the induction and maintenance of adaptive immune responses by altering or hijacking innate immune signaling pathways. What effect does this have on persistence of $B$. burgdorferi? The organized lymph node structure and the regulated migration of lymphocytes within secondary lymphoid tissues is crucial for effective interaction of $\mathrm{T}$ and $\mathrm{B}$ cells and the induction of adaptive immune effector mechanisms. Disruption of this structure is liable to have long-term negative consequences for the quality of the immune response and thus increases the likelihood of pathogen persistence. We outline below evidence to this effect.

\section{Interference of B. burgdorferi with GC Formation and Maintenance}

Activated B cells in the draining lymph nodes proliferate rapidly and initially form structures called extrafollicular foci (73). In extrafollicular foci, B cells become plasmablasts with or without $T$ cell help and produce large quantities of $B$. burgdorferi-specific antibodies rapidly. B cells in extrafollicular foci, however, do not undergo extensive affinity maturation, nor do they form memory B cells or long-lived plasma cells (115). For this, GCs are needed. GCs are a complex and highly organized structures that form within the $\mathrm{B}$ cell follicles of secondary lymphoid tissues that consist of GC B cells, CD4 T follicular helper cells $\left(\mathrm{T}_{\mathrm{FH}}\right)$, and follicular dendritic cells (FDCs). B cell activation and proliferation in GCs is facilitated by interaction with CD4 T cells and presentation of antigens on FDCs. High-frequency insertion of random mutations into the antigen-binding variable domain of the antibody molecule during this process, and the competition of the newly generated $\mathrm{B}$ cells for binding to antigen and for T cell-help, is thought to drive B cells with the highest affinity for antigen to outcompete other clones with weaker antigen binding. Although mechanisms are still unclear, GC responses lead to the development of two distinct cell populations: memory $\mathrm{B}$ cells, i.e., non-ASCs that circulate and can respond vigorously to repeat infection, and long-lived plasma cells, which continuously produce antibodies from their bone marrow niches and contribute to immune protection from reinfections (116-119). The GC environment also promotes CSR to IgG.

Despite the early presence of $B$. burgdorferi in the draining lymph nodes, $\mathrm{GC} B$ cell and $\mathrm{T}_{\mathrm{FH}}$ numbers remain low for the first 2 weeks in experimentally infected mice (74) and then become measurable. However, although live B. burgdorferi remained present in the lymph node for at least 1 year after infection, and thus, $B$. burgdorferi antigens were presumably continuously available, the GCs collapsed around 1 month after infection, and associated $B$ and $T$ cell numbers decreased steadily over the next month (69). As outlined earlier, the decline in the avidity of serum antibodies against Arp follows the collapse of GCs (69).

Consistent with the short-lived nature of the GC responses, their functional ineffectiveness was demonstrated by experiments showing a complete lack of memory recall responses to both $B$. burgdorferi antigens and a co-administered vaccine antigen for many months after infection with B. burgdorferi. Stable continued antibody production by long-lived ASCs in the bone marrow was also strongly delayed for at least 3 months after infection $(69,74)$. The delay in these important B cell response outcomes is especially dramatic considering a mouse's relatively short life span and likely frequent exposure in the wild. Although mice do not clear infection with $B$. burgdorferi, impairing the memory response could be advantageous to B. burgdorferi by leaving the host susceptible to secondary or superinfections. It might also prevent a timely and strong response to antigens that are dynamically upregulated and downregulated by $B$. burgdorferi as the infection progresses.

By decreasing the capacity of the host to produce effective antibodies against $B$. burgdorferi, the GC collapse may help $B$. burgdorferi evade clearance. The signals and mechanisms leading to the collapse, however, are unknown. One possible mechanism is the interference of B. burgdorferi with the complement system. Continued antigen presentation is crucial for hyperaffinity maturation, and components of the complement system are known to be involved in this process. Specifically, activated $\mathrm{C} 3$ and $\mathrm{C} 4$ fragments bound to antigen and adhere to complement receptors 1 and 2 (CR1 and CR2). These receptors are present on the major antigen-presenting cells in the GC, the FDC, and on GC B cells. It was shown previously that GCs will form normally in mice lacking CR1 and CR2, but collapse prematurely, before GCs can perform their important functions (120). This phenotype is strikingly similar to that seen in wild-type mice infected with $B$. burgdorferi. Interestingly, in $B$. burgdorferi-infected mice, although CR1 and CR2 are present on FDCs and GC B cells, C4 is not detectable (69). C4 is typically deposited on the surface of FDCs supporting antigen presentation. Interference with $\mathrm{C} 4$ deposition could inhibit antigen 
presentation by FDCs to GC B cells and thereby lead to GC collapse. B. burgdorferi interference with activation of complement could also have various indirect effects on GCs: changing the cytokine milieu, reducing antigen presentation to naïve $B$ cells via $\mathrm{CR} 1$ on APCs outside the GC, reducing naïve B cell activation via co-stimulation with CR2, and reducing opsonization (and thus uptake) of antigens. Exploring the role of complement and complement inhibition by B. burgdorferi during infection are important subjects for future studies.

\section{CONCLUSION}

The phenomena described earlier represent potential novel mechanism(s) for manipulation of the adaptive immune system by a pathogen that establishes persistent infections in its reservoir host. Elucidation of these mechanisms has important translational and clinical applications. A better understanding of how B. burgdorferi persists long term in rodents would be useful for understanding public health risks and devising appropriate preventative measures in endemic areas. Given the extensive similarities in the immune system of rodents and humans, it seems likely that the mechanisms of immune evasion and suppression outlined here may also be active in at least some infected humans. The induction of diseases such as carditis, arthritis, acrodermatitis chronica atrophicans, and neuroborreliosis seen in some patients with Lyme disease and infected companion animals, but rarely in mice, suggest

\section{REFERENCES}

1. Hofmeester TR, Coipan EC, van Wieren SE, Prins HHT, Takken W, Sprong H. Few vertebrate species dominate the Borrelia burgdorferi s.l. life cycle. Environ Res Lett (2016) 11(4):16. doi:10.1088/1748-9326/11/4/ 043001

2. Rudenko N, Golovchenko M, Grubhoffer L, Oliver JH. Updates on Borrelia burgdorferi sensu lato complex with respect to public health. Ticks Tick Borne Dis (2011) 2(3):123-8. doi:10.1016/j.ttbdis.2011.04.002

3. CDC (2016). Available from: http://www.cdc.gov

4. Margos G, Vollmer SA, Ogden NH, Fish D. Population genetics, taxonomy, phylogeny and evolution of Borrelia burgdorferi sensu lato. Infect Genet Evol (2011) 11(7):1545-63. doi:10.1016/j.meegid.2011.07.022

5. Brandt FC, Ertas B, Falk TM, Metze D, Boer-Auer A. Genotyping of Borrelia from formalin-fixed paraffin-embedded skin biopsies of cutaneous borreliosis and tick bite reactions by assays targeting the intergenic spacer region, ospA and ospC genes. Br J Dermatol (2014) 171(3):528-43. doi:10.1111/bjd.12855

6. Cook V, Barbour AG. Broad diversity of host responses of the white-footed mouse Peromyscus leucopus to Borrelia infection and antigens. Ticks Tick Borne Dis (2015) 6(5):549-58. doi:10.1016/j.ttbdis.2015.04.009

7. Barthold SW, Desouza MS, Janotka JL, Smith AL, Persing DH. Chronic Lyme borreliosis in the laboratory mouse. Am J Pathol (1993) 143(3):959-72.

8. Ma Y, Seiler KP, Eichwald EJ, Weis JH, Teuscher C, Weis JJ. Distinct characteristics of resistance to Borrelia burgdorferi-induced arthritis in C57BL/6N mice. Infect Immun (1998) 66(1):161-8.

9. Tilly K, Rosa PA, Stewart PE. Biology of infection with Borrelia burgdorferi. Infect Dis Clin North Am (2008) 22(2):217-34. doi:10.1016/j.idc. 2007.12.013

10. Voordouw MJ, Lachish S, Dolan MC. The Lyme disease pathogen has no effect on the survival of its rodent reservoir host. PLoS One (2015) 10(2):26. doi:10.1371/journal.pone.0118265

11. Radolf JD, Caimano MJ, Stevenson B, Hu LDT. Of ticks, mice and men: understanding the dual-host lifestyle of Lyme disease spirochaetes. Nat Rev Microbiol (2012) 10(2):87-99. doi:10.1038/nrmicro2714 maladaptation of B. burgdorferi to these hosts. Humans developing these inflammatory diseases to B. burgdorferi infection may have an immune system that is ineffectively suppressed by $B$. burgdorferi. There is good experimental evidence that a block of pro-inflammatory $\mathrm{T}$ cell responses, such as facilitated through blockade of IL-12, will cause reductions in arthritis development in $\mathrm{C} 3 \mathrm{H}$ mice, but it also causes increases in Borrelia tissue loads $(121,122)$. Development of therapeutics that can shift the balance toward immune activation and bacterial clearance without causing inflammation-induced diseases might provide superior tools to the current antibiotic therapies.

Much remains to be elucidated about the mechanisms by which Borrelia evades the host response. This area of research provides a particularly rich ground for collaboration among evolutionary biologists, ecologists, microbiologists, and immunologists.

\section{AUTHOR CONTRIBUTIONS}

KT and NB conceived of and wrote the article.

\section{FUNDING}

The work conducted in the Baumgarth lab and outlined in this review was supported by research support from the NIH/NIAID R01 AI073911, the Global Lyme Alliance, and the Steven \& Alexandra Cohen Foundation.

12. Persing DH, Mathiesen D, Podzorski D, Barthold SW. Genetic stability of Borrelia burgdorferi recovered from chronically infected immunocompetent mice. Infect Immun (1994) 62(8):3521-7.

13. Rego RO, Bestor A, Stefka J, Rosa PA. Population bottlenecks during the infectious cycle of the Lyme disease spirochete Borrelia burgdorferi. PLoS One (2014) 9(6):e101009. doi:10.1371/journal.pone.0101009

14. Hofmeister EK, Ellis BA, Glass GE, Childs JE. Longitudinal study of infection with Borrelia burgdorferi in a population of Peromyscus leucopus at a Lyme disease-enzootic site in Maryland. Am J Trop Med Hyg (1999) 60(4):598-609.

15. Bunikis J, Tsao J, Luke CJ, Luna MG, Fish D, Barbour AG. Borrelia burgdorferi infection in a natural population of Peromyscus leucopus mice: a longitudinal study in an area where lyme borreliosis is highly endemic. J Infect Dis (2004) 189(8):1515-23. doi:10.1086/382594

16. Olsen B, Gylfe A, Bergstrom S. Canary finches (Serinus canaria) as an avian infection model for Lyme borreliosis. Microb Pathog (1996) 20(6):319-24. doi:10.1006/mpat.1996.0030

17. Stanek G, Strle F. Lyme borreliosis. Lancet (2003) 362(9396):1639-47. doi:10.1016/s0140-6736(03)14798-8

18. Seifert SN, Khatchikian CE, Zhou W, Brisson D. Evolution and population genomics of the Lyme borreliosis pathogen, Borrelia burgdorferi. Trends Genet (2015) 31(4):201-7. doi:10.1016/j.tig.2015.02.006

19. Kurtenbach K, De Michelis S, Etti S, Schafer SM, Sewell HS, Brade V, et al. Host association of Borrelia burgdorferi sensu lato - the key role of host complement. Trends Microbiol (2002) 10(2):74-9. doi:10.1016/ s0966-842x(01)02298-3

20. Baum E, Hue F, Barbour AG. Experimental Infections of the Reservoir Species Peromyscus leucopus with Diverse Strains of Borrelia burgdorferi, a Lyme disease agent. MBio (2012) 3(6):11. doi:10.1128/mBio.00434-12

21. Zajac AJ, Blattman JN, Murali-Krishna K, Sourdive DJ, Suresh M, Altman $\mathrm{JD}$, et al. Viral immune evasion due to persistence of activated $\mathrm{T}$ cells without effector function. J Exp Med (1998) 188(12):2205-13. doi:10.1084/ jem.188.12.2205

22. Wherry EJ, Blattman JN, Murali-Krishna K, van der Most R, Ahmed R. Viral persistence alters CD8 T-cell immunodominance and tissue distribution 
and results in distinct stages of functional impairment. J Virol (2003) 77(8):4911-27. doi:10.1128/JVI.77.8.4911-4927.2003

23. Crawford A, Angelosanto JM, Kao C, Doering TA, Odorizzi PM, Barnett $\mathrm{BE}$, et al. Molecular and transcriptional basis of CD4(+) T cell dysfunction during chronic infection. Immunity (2014) 40(2):289-302. doi:10.1016/j. immuni.2014.01.005

24. McKinney EF, Lee JC, Jayne DR, Lyons PA, Smith KG. T-cell exhaustion, co-stimulation and clinical outcome in autoimmunity and infection. Nature (2015) 523(7562):612-6. doi:10.1038/nature14468

25. McKinney EF, Smith KG. T cell exhaustion and immune-mediated disease-the potential for therapeutic exhaustion. Curr Opin Immunol (2016) 43:74-80. doi:10.1016/j.coi.2016.09.005

26. Zuniga EI, Macal M, Lewis GM, Harker JA. Innate and adaptive immune regulation during chronic viral infections. Annu Rev Virol (2015) 2(1):573-97. doi:10.1146/annurev-virology-100114-055226

27. Anderson JF, Johnson RC, Magnarelli LA. Seasonal prevalence of Borrelia burgdorferi in natural populations of white-footed mice, Peromyscus leucopus. J Clin Microbiol (1987) 25(8):1564-6.

28. McLean RG, Ubico SR, Cooksey LM. Experimental infection of the eastern chipmunk (Tamias striatus) with the Lyme disease spirochete (Borrelia burgdorferi). J Wildl Dis (1993) 29(4):527-32. doi:10.7589/00903558-29.4.527

29. Appel MJG, Allan S, Jacobson RH, Lauderdale TL, Chang YF, Shin SJ, et al. Experimental Lyme disease in dogs produces arthritis and persistent infection. J Infect Dis (1993) 167(3):651-64. doi:10.1093/infdis/ 167.3.651

30. Roberts ED, Bohm RP, Cogswell FB, Lanners HN, Lowrie RC, Povinelli $\mathrm{L}$, et al. Chronic Lyme disease in the rhesus monkey. Lab Investig (1995) 72(2):146-60.

31. Embers ME, Barthold SW, Borda JT, Bowers L, Doyle L, Hodzic E, et al. Persistence of Borrelia burgdorferi in rhesus macaques following antibiotic treatment of disseminated infection. PLoS One (2012) 7(1):12. doi:10.1371/ journal.pone.0029914

32. Cook MJ. Lyme borreliosis: a review of data on transmission time after tick attachment. Int J Gen Med (2015) 8:1-8. doi:10.2147/IJGM.S73791

33. Kazimirova M, Stibraniova I. Tick salivary compounds: their role in modulation of host defences and pathogen transmission. Front Cell Infect Microbiol (2013) 3:43. doi:10.3389/fcimb.2013.00043

34. Kotal J, Langhansova H, Lieskovska J, Andersen JF, Francischetti IMB, Chavakis T, et al. Modulation of host immunity by tick saliva. J Proteomics (2015) 128:58-68. doi:10.1016/j.jprot.2015.07.005

35. Klein M, Bruhl TJ, Staudt V, Reuter S, Grebe N, Gerlitzki B, et al. Tick salivary sialostatin $\mathrm{L}$ represses the initiation of immune responses by targeting IRF4-dependent transcription in murine mast cells. J Immunol (2015) 195(2):621-31. doi:10.4049/jimmunol.1401823

36. Noelle RJ, Nowak EC. Cellular sources and immune functions of interleukin-9. Nat Rev Immunol (2010) 10(10):683-7. doi:10.1038/nri2848

37. Moretti S, Renga G, Oikonomou V, Galosi C, Pariano M, Iannitti RG, et al. A mast cell-ILC2-Th9 pathway promotes lung inflammation in cystic fibrosis. Nat Commun (2017) 8:14017. doi:10.1038/ncomms14017

38. Charon NW, Goldstein SF. Genetics of motility and chemotaxis of a fascinating group of bacteria: the spirochetes. Annu Rev Genet (2002) 36:47-73. doi:10.1146/annurev.genet.36.041602.134359

39. Moriarty TJ, Norman MU, Colarusso P, Bankhead T, Kubes P, Chaconas G. Real-time high resolution 3D imaging of the Lyme disease spirochete adhering to and escaping from the vasculature of a living host. PLoS Pathog (2008) 4(6):13. doi:10.1371/journal.ppat.1000090

40. Kenedy MR, Lenhart TR, Akins DR. The role of Borrelia burgdorferi outer surface proteins. FEMS Immunol Med Microbiol (2012) 66(1):1-19. doi:10.1111/j.1574-695X.2012.00980.x

41. Kline KA, Falker S, Dahlberg S, Normark S, Henriques-Normark B. Bacterial adhesins in host-microbe interactions. Cell Host Microbe (2009) 5(6):580-92. doi:10.1016/j.chom.2009.05.011

42. Ebady R, Niddam AF, Boczula AE, Kim YR, Gupta N, Tang TT, et al. Biomechanics of Borrelia burgdorferi vascular Interactions. Cell Rep (2016) 16(10):2593-604. doi:10.1016/j.celrep.2016.08.013

43. Caine JA, Coburn J. A short-term Borrelia burgdorferi infection model identifies tissue tropisms and bloodstream survival conferred by adhesion proteins. Infect Immun (2015) 83(8):3184-94. doi:10.1128/iai.00349-15
44. Lin YP, Chen Q, Ritchie JA, Dufour NP, Fischer JR, Coburn J, et al. Glycosaminoglycan binding by Borrelia burgdorferi adhesin BBK32 specifically and uniquely promotes joint colonization. Cell Microbiol (2015) 17(6):860-75. doi:10.1111/cmi.12407

45. Garcia BL, Zhi H, Wager B, Hook M, Skare JT. Borrelia burgdorferi BBK32 inhibits the classical pathway by blocking activation of the $\mathrm{C} 1$ complement complex. PLoS Pathog (2016) 12(1):1-28. doi:10.1371/journal.ppat. 1005404

46. Kumar D, Ristow LC, Shi M, Mukherjee P, Caine JA, Lee W-Y, et al. Intravital imaging of vascular transmigration by the lyme spirochete: requirement for the integrin binding residues of the B. burgdorferi P66 protein. PLoS Pathog (2015) 11(12):e1005333. doi:10.1371/journal. ppat. 1005333

47. Shi YL, Xu QL, Seemanaplli SV, McShan K, Liang FT. Common and unique contributions of decorin-binding proteins $\mathrm{A}$ and $\mathrm{B}$ to the overall virulence of Borrelia burgdorferi. PLoS One (2008) 3(10):11. doi:10.1371/ journal.pone. 0003340

48. Liang FT, Brown EL, Wang T, Lozzo RV, Fikrig E. Protective niche for Borrelia burgdorferi to evade humoral immunity. Am J Pathol (2004) 165(3):977-85. doi:10.1016/s0002-9440(10)63359-7

49. Coburn J, Leong J, Chaconas G. Illuminating the roles of the Borrelia burgdorferi adhesins. Trends Microbiol (2013) 21(8):372-9. doi:10.1016/j. tim.2013.06.005

50. Brissette CA, Gaultney RA. That's my story, and I'm sticking to it-an update on B. burgdorferi adhesins. Front Cell Infect Microbiol (2014) 4:13. doi:10.3389/fcimb.2014.00041

51. Imai DM, Feng SL, Hodzic E, Barthold SW. Dynamics of connective-tissue localization during chronic Borrelia burgdorferi infection. Lab Investig (2013) 93(8):900-10. doi:10.1038/labinvest.2013.81

52. Coleman JL, Roemer EJ, Benach JL. Plasmin-coated Borrelia burgdorferi degrades soluble and insoluble components of the mammalian extracellular matrix. Infect Immun (1999) 67(8):3929-36.

53. Barthel D, Schindler S, Zipfel PF. Plasminogen is a complement inhibitor. J Biol Chem (2012) 287(22):18831-42. doi:10.1074/jbc.M111.323287

54. Gebbia JA, Coleman JL, Benach JL. Selective induction of matrix metalloproteinases by Borrelia burgdorferi via toll-like receptor 2 in monocytes. $J$ Infect Dis (2004) 189(1):113-9. doi:10.1086/380414

55. Zhao ZH, Chang H, Trevino RP, Whren K, Bhawan J, Klempner MS. Selective up-regulation of matrix metalloproteinase-9 expression in human erythema migrans skin lesions of acute Lyme disease. J Infect Dis (2003) 188(8):1098-104. doi:10.1086/379039

56. Heilpern AJ, Wertheim W, He J, Perides G, Bronson RT, Hu LT. Matrix metalloproteinase 9 plays a key role in Lyme arthritis but not in dissemination of Borrelia burgdorferi. Infect Immun (2009) 77(7):2643-9. doi:10.1128/ Iai.00214-09

57. Blom AM, Hallstrom T, Riesbeck K. Complement evasion strategies of pathogens-acquisition of inhibitors and beyond. Mol Immunol (2009) 46(14):2808-17. doi:10.1016/j.molimm.2009.04.025

58. Laarman A, Milder F, van Strijp J, Rooijakkers S. Complement inhibition by gram-positive pathogens: molecular mechanisms and therapeutic implications. J Mol Med (Berl) (2010) 88(2):115-20. doi:10.1007/ s00109-009-0572-y

59. Ricklin D. Manipulating the mediator: modulation of the alternative complement pathway C3 convertase in health, disease and therapy. Immunobiology (2012) 217(11):1057-66. doi:10.1016/j.imbio.2012.07.016

60. de Taeye SW, Kreuk L, van Dam AP, Hovius JW, Schuijt TJ. Complement evasion by Borrelia burgdorferi: it takes three to tango. Trends Parasitol (2013) 29(3):119-28. doi:10.1016/j.pt.2012.12.001

61. Merle NS, Church SE, Fremeaux-Bacchi V, Roumenina LT. Complement system part I - molecular mechanisms of activation and regulation. Front Immunol (2015) 6:262. doi:10.3389/fimmu.2015.00262

62. Kraiczy P. Hide and seek: how lyme disease spirochetes overcome complement attack. Front Immunol (2016) 7:8. doi:10.3389/fimmu. 2016.00385

63. Bykowski T, Woodman ME, Cooley AE, Brissette CA, Wallich R, Brade $\mathrm{V}$, et al. Borrelia burgdorferi complement regulator-acquiring surface proteins (BbCRASPs): expression patterns during the mammal-tick infection cycle. Int J Med Microbiol (2008) 298:249-56. doi:10.1016/j.ijmm.2007. 10.002 
64. Pietikainen J, Meri T, Blom AM, Meri S. Binding of the complement inhibitor C4b-binding protein to Lyme disease borreliae. Mol Immunol (2010) 47(6):1299-305. doi:10.1016/j.molimm.2009.11.028

65. Barthold SW, Bockenstedt LK. Passive immunizing activity of sera from mice infected with Borrelia burgdorferi. Infect Immun (1993) 61(11): 4696-702.

66. Barthold SW. Specificity of infection-induced immunity among Borrelia burgdorferi sensu late species. Infect Immun (1999) 67(1):36-42.

67. Barbour AG, Jasinskas A, Kayala MA, Davies DH, Steere AC, Baldi P, et al. A genome-wide proteome array reveals a limited set of immunogens in natural infections of humans and white-footed mice with Borrelia burgdorferi. Infect Immun (2008) 76(8):3374-89. doi:10.1128/iai.00048-08

68. Hodzic E, Feng SL, Freet KJ, Barthold SW. Borrelia burgdorferi population dynamics and prototype gene expression during infection of immunocompetent and immunodeficient mice. Infect Immun (2003) 71(9):5042-55. doi:10.1128/iai.71.9.5042-5055.2003

69. Elsner RA, Hastey CJ, Olsen KJ, Baumgarth N. Suppression of long-lived humoral immunity following Borrelia burgdorferi infection. PLoS Pathog (2015) 11(7):19. doi:10.1371/journal.ppat.1004976

70. McKisic MD, Barthold SW. T-Cell-independent responses to Borrelia burgdorferi are critical for protective immunity and resolution of lyme disease. Infect Immun (2000) 68(9):5190-7. doi:10.1128/iai.68.9.5190-5197.2000

71. McKisic MD, Redmond WL, Barthold SW. T cell-mediated pathology in murine lyme borreliosis. J Immunol (2000) 164(12):6096-9. doi:10.4049/ jimmunol.164.12.6096

72. Collins AM. IgG subclass co-expression brings harmony to the quartet model of murine IgG function. Immunol Cell Biol (2016) 94(10):949-54. doi:10.1038/icb.2016.65

73. Tunev SS, Hastey CJ, Hodzic E, Feng SL, Barthold SW, Baumgarth N. Lymphoadenopathy during lyme borreliosis is caused by spirochete migration-induced specific B cell activation. PLoS Pathog (2011) 7(5):14. doi:10.1371/journal.ppat.1002066

74. Hastey CJ, Elsner RA, Barthold SW, Baumgarth N. delays and diversions mark the development of B cell responses to Borrelia burgdorferi infection. J Immunol (2012) 188(11):5612-22. doi:10.4049/jimmunol.1103735

75. Elsner RA, Hastey CJ, Baumgarth N. CD4(+) T cells promote antibody production but not sustained affinity maturation during Borrelia burgdorferi infection. Infect Immun (2015) 83(1):48-56. doi:10.1128/ Iai.02471-14

76. Embers ME, Alvarez X, Ooms T, Philipp MT. The failure of immune response evasion by linear plasmid 28-1-deficient Borrelia burgdorferi is attributable to persistent expression of an outer surface protein. Infect Immun (2008) 76(9):3984-91. doi:10.1128/iai.00387-08

77. Rogovskyy AS, Casselli T, Tourand Y, Jones CR, Owen JP, Mason KL, et al. Evaluation of the importance of VlsE antigenic variation for the enzootic cycle of Borrelia burgdorferi. PLoS One (2015) 10(4):21. doi:10.1371/journal. pone. 0124268

78. Hefty PS, Jolliff SE, Caimano MJ, Wikel SK, Radolf JD, Akins DR. Regulation of OspE-related, OspF-related, and Elp lipoproteins of Borrelia burgdorferi strain 297 by mammalian host-specific signals. Infect Immun (2001) 69(6):3618-27. doi:10.1128/iai.69.6.3618-3627.2001

79. Iyer R, Caimano MJ, Luthra A, Axline D, Corona A, Iacobas DA, et al. Stage-specific global alterations in the transcriptomes of Lyme disease spirochetes during tick feeding and following mammalian host adaptation. Mol Microbiol (2015) 95(3):509-38. doi:10.1111/mmi.12882

80. Liang FT, Nelson FK, Fikrig E. Molecular adaptation of Borrelia burgdorferi in the murine host. J Exp Med (2002) 196(2):275-80. doi:10.1084/ jem. 20020770

81. Gilmore RD, Kappel KJ, Dolan MC, Burkot TR, Johnson BJB. Outer surface protein $\mathrm{C}(\mathrm{OspC})$, but not $\mathrm{P} 39$, is a protective immunogen against a tick-transmitted Borrelia burgdorferi challenge: evidence for a conformational protective epitope in OspC. Infect Immun (1996) 64(6):2234-9.

82. Mbow ML, Gilmore RD, Titus RG. An OspC-specific monoclonal antibody passively protects mice from tick-transmitted infection by Borrelia burgdorferi B31. Infect Immun (1999) 67(10):5470-2.

83. Grimm D, Tilly K, Byram R, Stewart PE, Krum JG, Bueschel DM, et al. Outer-surface protein $\mathrm{C}$ of the Lyme disease spirochete: a protein induced in ticks for infection of mammals. Proc Natl Acad Sci U S A (2004) 101(9):3142-7. doi:10.1073/pnas.0306845101
84. Tilly K, Krum JG, Bestor A, Jewett MW, Grimm D, Bueschel D, et al. Borrelia burgdorferi OspC protein required exclusively in a crucial early stage of mammalian infection. Infect Immun (2006) 74(6):3554-64. doi:10.1128/ iai.01950-05

85. Liang FT, Jacobs MB, Bowers LC, Philipp MT. An immune evasion mechanism for spirochetal persistence in Lyme borreliosis. J Exp Med (2002) 195(4):415-22. doi:10.1084/jem.20011870

86. Barbour AG, Restrepo BI. Antigenic variation in vector-borne pathogens. Emerg Infect Dis (2000) 6(5):449-57. doi:10.3201/eid0605.000502

87. Palmer GH, Bankhead T, Lukehart SA. 'Nothing is permanent but change' - antigenic variation in persistent bacterial pathogens. Cell Microbiol (2009) 11(12):1697-705. doi:10.1111/j.1462-5822.2009.01366.x

88. Raffel SJ, Battisti JM, Fischer RJ, Schwan TG. Inactivation of genes for antigenic variation in the relapsing fever spirochete Borrelia hermsii reduces infectivity in mice and transmission by ticks. PLoS Pathog (2014) 10(4):e1004056. doi:10.1371/journal.ppat.1004056

89. Norris SJ. vls antigenic variation systems of lyme disease Borrelia: eluding host immunity through both random, segmental gene conversion and framework heterogeneity. Microbiol Spectr (2014) 2(6):1-18. doi:10.1128/ microbiolspec.MDNA3-0038-2014

90. Lawrenz MB, Wooten RM, Norris SJ. Effects of vlsE complementation on the infectivity of Borrelia burgdorferi lacking the linear plasmid lp28-1. Infect Immun (2004) 72(11):6577-85. doi:10.1128/iai.72.11.65776585.2004

91. Bankhead T, Chaconas G. The role of VlsE antigenic variation in the Lyme disease spirochete: persistence through a mechanism that differs from other pathogens. Mol Microbiol (2007) 65(6):1547-58. doi:10.1111/j.1365-2958.2007.05895.x

92. Rogovskyy AS, Bankhead T. Variable VlsE is critical for host reinfection by the lyme disease spirochete. PLoS One (2013) 8(4):12. doi:10.1371/journal. pone.0061226

93. Zhang JR, Norris SJ. Kinetics and in vivo induction of genetic variation of vlsE in Borrelia burgdorferi. Infect Immun (1998) 66(8):3689-97.

94. Johnson PLF, Kochin BF, Ahmed R, Antia R. How do antigenically varying pathogens avoid cross-reactive responses to invariant antigens? Proc Biol Sci (2012) 279(1739):2777-85. doi:10.1098/rspb.2012.0005

95. Barbour AG, Travinsky B. Evolution and distribution of the ospC gene, a transferable serotype determinant of Borrelia burgdorferi. MBio (2010) 1(4):11. doi:10.1128/mBio.00153-10

96. Casjens SR, Mongodin EF, Qiu WG, Luft BJ, Schutzer SE, Gilcrease EB, et al. Genome stability of lyme disease spirochetes: comparative genomics of Borrelia burgdorferi plasmids. PLoS One (2012) 7(3):24. doi:10.1371/ journal.pone.0033280

97. Di L, Pagan PE, Packer D, Martin CL, Akther S, Ramrattan G, et al. BorreliaBase: a phylogeny-centered browser of Borrelia genomes. BMC Bioinformatics (2014) 15:11. doi:10.1186/1471-2105-15-233

98. Cerar T, Strle F, Stupica D, Ruzic-Sabljic E, McHugh G, Steere AC, et al. Differences in genotype, clinical features, and inflammatory potential of Borrelia burgdorferi sensu stricto strains from Europe and the United States. Emerg Infect Dis (2016) 22(5):818-27. doi:10.3201/eid2205.151806

99. Elias AF, Stewart PE, Grimm D, Caimano MJ, Eggers CH, Tilly K, et al. Clonal polymorphism of Borrelia burgdorferi strain B31 MI: implications for mutagenesis in an infectious strain background. Infect Immun (2002) 70(4):2139-50. doi:10.1128/IAI.70.4.2139-2150.2002

100. Rogovskyy AS, Bankhead T. Bacterial heterogeneity is a requirement for host superinfection by the Lyme disease spirochete. Infect Immun (2014) 82(11):4542-52. doi:10.1128/iai.01817-14

101. Piesman J, Dolan MC, Happ CM, Luft BJ, Rooney SE, Mather TN, et al. Duration of immunity to reinfection with tick-transmitted Borrelia burgdorferi in naturally infected mice. Infect Immun (1997) 65(10): 4043-7.

102. Slifka MK, Ahmed R. Long-term antibody production is sustained by antibody-secreting cells in the bone marrow following acute viral infection. Ann N Y Acad Sci (1996) 797:166-76. doi:10.1111/j.1749-6632.1996. tb52958.x

103. Halliley JL, Tipton CM, Liesveld J, Rosenberg AF, Darce J, Gregoretti IV, et al. Long-lived plasma cells are contained within the CD19(-)CD38(hi) CD138(+) subset in human bone marrow. Immunity (2015) 43(1):132-45. doi:10.1016/j.immuni.2015.06.016 
104. Binder S, Telschow A, Meyer-Hermann M. Population dynamics of Borrelia burgdorferi in Lyme disease. Front Microbiol (2012) 3(104):104. doi:10.3389/fmicb.2012.00104

105. Imai DM, Samuels DS, Feng SL, Hodzic E, Olsen K, Barthold SW. The early dissemination defect attributed to disruption of decorin-binding proteins is abolished in chronic murine lyme borreliosis. Infect Immun (2013) 81(5):1663-73. doi:10.1128/iai.01359-12

106. Griffin AJ, Li LX, Voedisch S, Pabst O, McSorley SJ. Dissemination of persistent intestinal bacteria via the mesenteric lymph nodes causes typhoid relapse. Infect Immun (2011) 79(4):1479-88. doi:10.1128/iai.01033-10

107. Gonzalez RJ, Weening EH, Frothingham R, Sempowski GD, Miller VL. Bioluminescence imaging to track bacterial dissemination of Yersinia pestis using different routes of infection in mice. BMC Microbiol (2012) 12:12. doi:10.1186/1471-2180-12-147

108. Lynskey NN, Banerji S, Johnson LA, Holder KA, Reglinski M, Wing PAC, et al. Rapid lymphatic dissemination of encapsulated group a streptococci via lymphatic vessel endothelial receptor-1 interaction. PLoS Pathog (2015) 11(9):19. doi:10.1371/journal.ppat.1005137

109. Yegutkin GG, Hytonen J, Samburski SS, Yrjanainen H, Jalkanen S, Viljanen MK. disordered lymphoid purine metabolism contributes to the pathogenesis of persistent Borrelia garinii infection in mice. J Immunol (2010) 184(9):5112-20. doi:10.4049/jimmunol.0902760

110. Hastey CJ, Ochoa J, Olsen KJ, Barthold SW, Baumgarth N. MyD88-and TRIF-independent induction of type I interferon drives naive $\mathrm{b}$ cell accumulation but not loss of lymph node architecture in Lyme disease. Infect Immun (2014) 82(4):1548-58. doi:10.1128/iai.00969-13

111. Achtman AH, Khan M, MacLennan IC, Langhorne J. Plasmodium chabaudi chabaudi infection in mice induces strong B cell responses and striking but temporary changes in splenic cell distribution. J Immunol (2003) 171(1):317-24. doi:10.4049/jimmunol.171.1.317

112. St John AL, Abraham SN. Salmonella disrupts lymph node architecture by TLR4-mediated suppression of homeostatic chemokines. Nat Med (2009) 15(11):1259-65. doi:10.1038/nm.2036

113. Steere AC. Lyme disease. New Engl J Med (1989) 321(9):586-96. doi:10.1056/ nejm198908313210906

114. Summers BA, Straubinger AF, Jacobson RH, Chang YT, Appel MJG, Straubinger RK. Histopathological studies of experimental Lyme disease in the dog. J Comp Pathol (2005) 133(1):1-13. doi:10.1016/j.jcpa.2004.11.006

115. MacLennan IC, Toellner KM, Cunningham AF, Serre K, Sze DM, Zuniga E, et al. Extrafollicular antibody responses. Immunol Rev (2003) 194:8-18. doi:10.1034/j.1600-065X.2003.00058.x
116. MacLennan IC. Germinal centers. Annu Rev Immunol (1994) 12:117-39. doi:10.1146/annurev.iy.12.040194.001001

117. Shlomchik MJ, Weisel F. Germinal centers. Immunol Rev (2012) 247:5-10. doi:10.1111/j.1600-065X.2012.01125.x

118. Shlomchik MJ, Weisel F. Germinal center selection and the development of memory B and plasma cells. Immunol Rev (2012) 247(1):52-63. doi:10.1111/j.1600-065X.2012.01124.x

119. Zotos D, Tarlinton DM. Determining germinal centre B cell fate. Trends Immunol (2012) 33(6):281-8. doi:10.1016/j.it.2012.04.003

120. Donius LR, Weis JJ, Weis JH. Murine complement receptor 1 is required for germinal center B cell maintenance but not initiation. Immunobiology (2014) 219(6):440-9. doi:10.1016/j.imbio.2014.02.007

121. Anguita J, Persing DH, Rincon M, Barthold SW, Fikrig E. Effect of anti-interleukin 12 treatment on murine lyme borreliosis. J Clin Invest (1996) 97(4):1028-34. doi:10.1172/JCI118494

122. Bockenstedt LK, Kang I, Chang C, Persing D, Hayday A, Barthold SW. CD4+ T helper 1 cells facilitate regression of murine Lyme carditis. Infect Immun (2001) 69(9):5264-9. doi:10.1128/IAI.69.9.5264-5269.2001

123. Moriarty TJ, Norman MU, Colarusso P, Bankhead T, Kubes P, Chaconas G. Real-time high resolution 3D imaging of the Lyme disease spirochete adhering to and escaping from the vasculature of a living host. PLoS Pathog (2008) 4(6):e1000090. doi:10.1371/journal.ppat.1000090

124. Tsao JI. Reviewing molecular adaptations of lyme borreliosis spirochetes in the context of reproductive fitness in natural transmission cycles. Vet Res (2009) 40(2):42. doi:10.1051/vetres/2009019

125. Wikel S. Ticks and tick-borne pathogens at the cutaneous interface: host defenses, tick countermeasures, and a suitable environment for pathogen establishment. Front. Microbiol (2013) 4:10. doi:10.3389/ fmicb.2013.00337

Conflict of Interest Statement: The authors declare that the research was conducted in the absence of any commercial or financial relationships that could be construed as a potential conflict of interest.

Copyright (c) 2017 Tracy and Baumgarth. This is an open-access article distributed under the terms of the Creative Commons Attribution License (CC BY). The use, distribution or reproduction in other forums is permitted, provided the original author(s) or licensor are credited and that the original publication in this journal is cited, in accordance with accepted academic practice. No use, distribution or reproduction is permitted which does not comply with these terms. 